\title{
Hydatid Disease during Pregnancy, the Challenging Management: Case Report and Review of Literature
}

\author{
Janoub Khazaal* \\ Associate Professor, Faculty of Medical Sciences, University of Lebanese, Lebanon \\ ${ }^{\star}$ Corresponding author: Janoub Khazaal, Associate Professor, Faculty of Medical Sciences, University of Lebanese, Lebanon
}

Received: August 16, 2021; Accepted: August 23, 2021; Published: August 30, 2021

\begin{abstract}
Human echinococcosis is a parasitic infestation of human by a tapeworm called Echinococcus granulosus. It can affect pregnant women at a low incidence. Mainly asymptomatic, it has a variety of presentations. Its main location is in the liver. Hydatid disease requires multidisciplinary care especially in pregnant patients. It can be treated medically or surgically according to each case.

We present a case of 27-year-old woman, G4 P3 with three previous cesarean sections, known to have hydatid cyst disease, presenting at 32 weeks of gestation, complaining of intermittent crampy right upper quadrant abdominal pain. Abdominal ultrasound done at 11 weeks of gestation, showed hepatomegaly along with a $9 \mathrm{~cm} \times 6 \mathrm{~cm}$ mass suggestive of hydatid cyst that was followed by ultrasounds frequently. The patient was followed by a multidisciplinary team when she was then started on Albendazol $400 \mathrm{mg}$ twice daily starting 32 weeks until delivery, then for two days post operatively. She did well and was discharged home in good condition.

Hydatid disease has a special consideration when associated with pregnancy. Many cases have been reported in literature yet only few reviews of literature and one meta-analysis have been reported. Management decision is quite meticulous. It depends on cystic nature and activity on one side and on the clinical picture of the patient on the other side. In all cases, cesarean section is advised. In spite of all recommendations, further prospective studies using larger samples are needed for clear-cut algorithmic guidelines for hydatid disease management in pregnancy.
\end{abstract}

\section{Introduction}

Human echinococcosis, or hydatid disease, also known as "cyst full of water" as described by Hippocrates, is a parasitic infestation of human by a tapeworm called Echinococcus granulosus [1-4]. This disease can be seen in all population including pregnant women $[5,6]$. Despite that, it is currently classified among the most neglected parasitic disease [1,5]. Hydatid cyst has a variety of presentations. It may remain asymptomatic for many years and may be discovered incidentally by radiography $[3,4]$. The most common site of hydatid cyst is the liver $[4,6]$. It might be found also in any part of the body including lungs, bones and soft tissues [3,5,7]. Hydatid cyst in pregnancy is rare, and usually affects the liver $[3,6]$. Its diagnosis in pregnancy might not be difficult, but the management is problematic [4]. It requires multidisciplinary care [2]. Both medical and surgical treatment are available but each case should be individualized due to the limited experience, lack of standardized consensus and paucity of information in the literature as only data from case reports are available [6]. For that, obstetricians should be more aware of the clinical features, diagnosis and treatment of this unusual disease [2].

Here we present a case of a pregnant woman with a huge liver hydatid disease.

\section{Case Presentation}

A 27-year-old woman G4 P3 with previous three cesarean sections, known to have hydatid cyst disease, presented to the outpatient department at 32 weeks of gestation with an intermittent crampy right upper quadrant abdominal pain, not related to oral intake, not relieved by any position, and not associated with any nausea or vomiting, anorexia, jaundice, or pruritis. She was diagnosed recently with recurrent hydatid cyst disease during pregnancy, for which she was referred to "high risk pregnancies clinic" [8-10].

Her history of hydatid disease goes back to her second pregnancy, at 6 weeks gestational age; when she underwent a laparotomy for excision of hepatic hydatid cyst, after which she didn't receive any medical treatment. Four years later, the patient had recurrent disease which was managed by cystectomy in segments IV and VII of the liver in addition to lung cystectomy. Post operatively, the patient received Albendazole that was continued for 2 months.

Beside the hydatid disease, she was having a smooth course of pregnancy. During this pregnancy the patient was followed at an outside clinic, where serology of Echinococcus granulosus (IgG and IgM) was requested in the first trimester and came back negative. Serial abdominal ultrasounds were done. The first one, done at 11 weeks of gestation, showed a $9 \mathrm{~cm} \times 6 \mathrm{~cm}$ lobulated cystic formation at the level of segment IV of the liver and having small wall calcifications. Repeated at $13+6$ weeks of gestation, it showed: two contiguous lobulated cystic structures, at the level of the dome of the liver. The first cyst in segment VIII was extending to segment IV: $3.4 \mathrm{~cm}$ in transverse diameter, demonstrating peripheral hyperechoic rim that was not associated with post attenuation and most likely 
not corresponding to calcification. In addition, hyperechoic content was identified in the first cyst corresponding to post enhancement. It was concluded that the hyperechoic content could represent hydatid sand, and thus, this cystic structure in the context of hydatid disease was classified as CE1 according to WHO classification. The presence of a larger cyst with lobulated contours in segment VII and VIII was also noted measuring $6.5 \mathrm{~cm} \times 6.5 \mathrm{~cm}$ with identification of anechoic content and few septations in the periphery particularly at its posterior aspect. The clear pattern of daughter cyst could not be identified thus a CE2 cyst couldn't be suggested with certainty. The third US, done at 22 weeks of gestation, showed that the largest cyst previously seen in segment VIII was stable in size, demonstrating internal septations with identification of daughter cyst-like structures posteriorly. Moreover, when compared to previous report, the other lobulated cyst in segment VIII and IV having lobulated contours increased in size to $5 \times 3.4 \mathrm{~cm}$ (compared to $3.4 \times 3.2 \mathrm{~cm}$ ). It was still showing a sand-like hyperechoic content, and demonstrating peripheral hyperechoic rim with posterior enhancement. The liver was enlarged to $17 \mathrm{~cm}$ in size.

Upon presentation, the patient had unremarkable vital signs. Her physical exam was limited due to obesity. It showed, however, right subcostal incision scar upon inspection, hepatomegaly with dullness but soft and non-tender abdomen.

The case was discussed by the multidisciplinary team members including the obstetrician, the maternal medicine, and the infectious diseases specialists. A decision to start Albendazol $400 \mathrm{mg}$ twice daily till delivery (for three) weeks, was taken. Abdominal ultrasound was repeated just before cesarean section that showed three liver hydatid cysts, one of them decreasing in size from $6 \mathrm{~cm}$ to $5 \mathrm{~cm}$, and two other stable cysts of $3 \mathrm{~cm}$. Nevertheless, we couldn't confirm if the cysts are stable or in active phase. There was no indication to operate those cysts at the time of C-section.

The patient continued medical treatment till cesarean delivery scheduled at 37 weeks; and for two days post operatively. She was did well post op and was discharged home on day two postop. The patient was seen one-week thereafter and was doing well. She continued to be followed by ID team and was planned to repeat abdominal US 6 months after delivery.

\section{Discussion}

As previously mentioned hydatid cyst disease is a parasitic infestation by a tapeworm most commonly by Echinococcus granulosus [10-15]. Contaminated food and water by feces of definitive infective host or poor hygiene in infestation areas are the main roots of exposure. The occurrence of this disease in pregnancy is low with a prevalence of $1 / 20,000$ to $1 / 30,000$.

Hydatid disease is mostly asymptomatic [10] but affected individuals can present with abdominal pain, abdominal mass or vague abdominal symptoms $[11,14]$ which may mimic the usual symptoms occurring in pregnancy.

It is important to note that blood tests are nonspecific. Many serologic tests are available mainly IgG ELISA test and Indirect Hemagglutination Test $[10,14]$, yet negative tests cannot rule out the disease.

Ultrasound is the primary diagnostic imaging tool. However, it cannot distinguish a benign cystic form from an abscess $[9,11]$. CT scan is the best imaging modality, being able to determine accurately the anatomical location and assess other $[9,11,14]$.

There are five subtypes of cystic echinococcosis according to the WHO and Gharbi classification systems (Table 1).

There are three management modalities depending on the activity of the cyst [11]. The first one is the surgical management requires opening the cyst, injecting a solicidal agent, evacuating the cyst and pericystectomy. It is best used for ruptured cysts, cysts with biliary fistulae, cysts compressing vital structures, cysts with secondary infection or hemorrhage, or cysts with multiple daughter cysts $[11,14]$. Other indications include cysts larger than 10 centimeters and superficial cysts which carry a risk of rupture [14]. Preoperative and postoperative use of Albendazole decreases the viability of cysts at the time of surgery and significantly reduces the chances of cyst recurrence. Albendazole for three 28 -day courses of $10 \mathrm{mg} / \mathrm{kg} /$ day in divided doses separated by 2 -week intervals post operatively is usually used [11-14]. Surgical management carries 2-25\% risk of recurrence.

The second therapeutic modality is the percutaneous treatment is based on destroying the germinal layer with a solicidal agent, a process that requires puncture, aspiration, injection and reaspiration $[11,14]$. This method is done under CT or ultrasound guidance for CE1 and CE3-A cysts which do not contain daughter cysts [11,14]. It is avoided in complicated or superficial cysts due to the risk of rupture [11]. The last modality is the medical treatment based on Albendazole administration.

Hydatid disease has a special consideration when associated with pregnancy. Many cases have been reported in literature yet only few reviews of literature and one meta-analysis have been reported.

Table 1: WHO and Gabri classification system for cystic echinococcosis cysts.

\begin{tabular}{|l|l|l|l|}
\hline \multicolumn{1}{|c|}{ WHO-IWGE 2001 } & \multicolumn{1}{|c|}{ Gharbi 1981 } & \multicolumn{1}{c|}{ Description } \\
\hline CE1 & Type I & Unilocular unechoic cystic lesion with double line sign \\
\hline CE2 & Type III & Multiseptated, "rpsette-like" "honeycomb cyst \\
\hline CE3 A & Type II & Cyst with detached membranes (water-lily-sign) & Active \\
\hline CE3 B & Type III & Cyst with daughter cysts in solid matrix \\
\hline CE4 & Type IV & Cyst with heterogenous hypoechoic/hyperechoic contents. No daughter cysts \\
\hline CE5 & Type V & Solid cyst with calcified wall & Transitional \\
\hline
\end{tabular}


Hydatid disease in pregnancy is a rare condition occurring in parts of the world. It is usually diagnosed incidentally during an obstetrical ultrasound [8].

A pregnant patient may complain of abdominal discomfort and pain in the right upper quadrant area. In large cysts cases, nausea, vomiting and early satiety may be felt due to a mass effect [8].

The main challenge in pregnancy is in regards to treatment modality which is affected by cyst classification. Types 1 and 2 are usually treated by ultrasound guided percutaneous aspiration [8]. Types 3 and 4 , with the exception of calcified cysts are mainly surgically treated [8]. However, due to scarcity of data, there is no standardized approach. Below, an algorithm will be stated for managing hydatid disease in pregnancy based on the only meta-analysis published on 2018 (Figure 1) [8].

The main medical therapy for echinococcal cysts is based on Benzimidazole, namely Albendazole. It acts on inhibiting glucose uptake by the parasite, thus generating metabolic and structural alteration leading to parasitic death. It is categorized as category $\mathrm{C}$ by the FDA [8]. Thus, it is not recommended for use in first trimester $[8,14]$, yet it can be used afterwards.

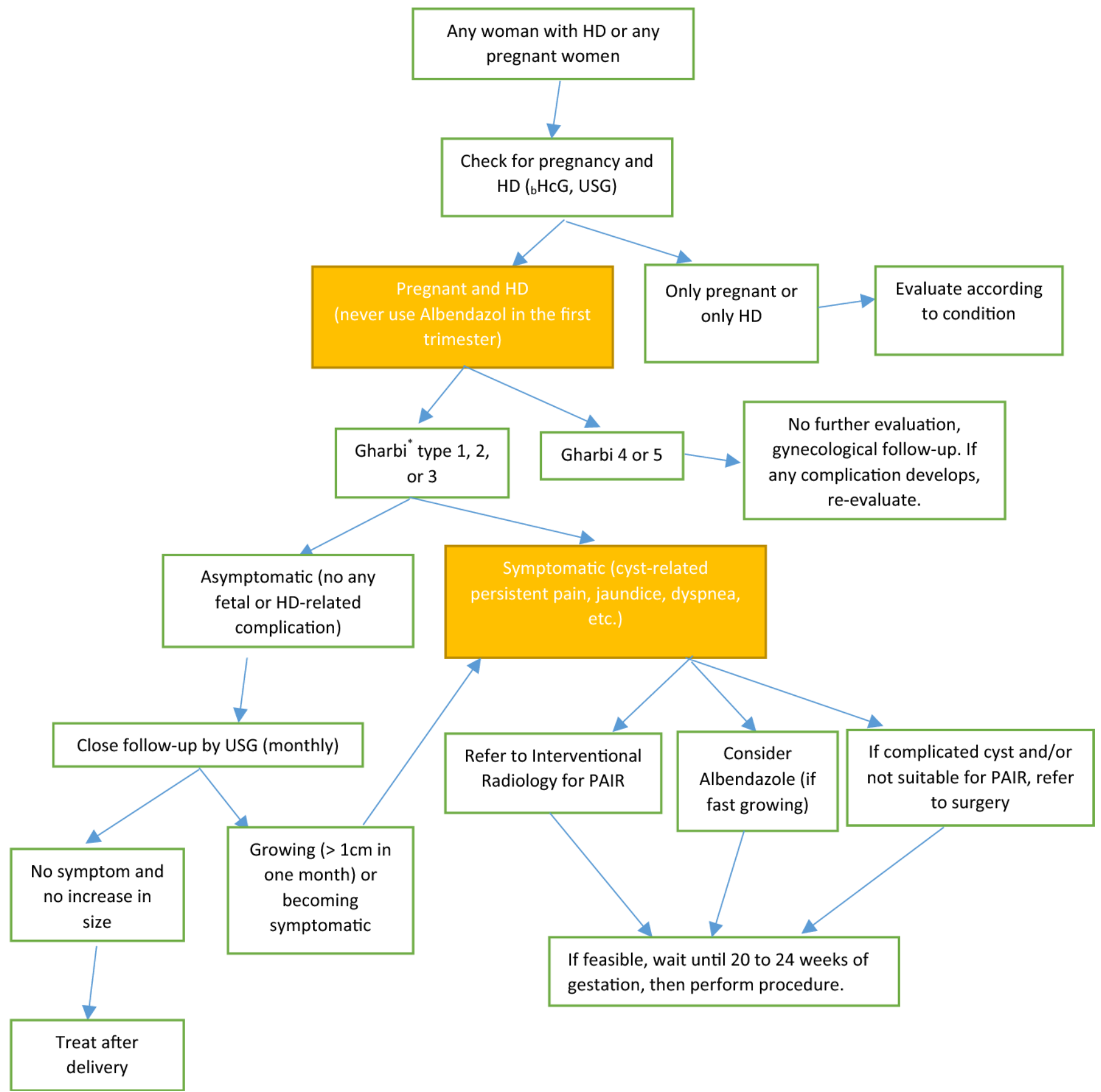

Figure 1: Approach and treatment algorithm for pregnancy complicated with Hidatid disease. HD Hydatid Disease; b HCG beta-human chorionic gonadotropin;"Ultrasound-based classification of cyst hydatid according to Gharbi et al.; PAIR percutaneous aspiration irrigation and re-aspiration (8).

Celik, S. et al. Archives of Gynecology and Obstetrics 298 (2018): 103-110. 
According to Celik et al. a thin walled, large, peripherally located cyst in the liver is more likely to rupture than a small, centrally located cyst. Therefore, women with cysts characterized as at high risk of rupture should be referred at some point for intervention (surgery or PAIR) [8]. If no intervention is to be performed, then delivery by cesarean section is preferred. In optimal condition, a monthly close follow up is recommended. If conditions are worsened, intervention is considered after 20-24 weeks of gestation to allow for fetal maturation [8].

Concerning our patient, she started Albendazole $400 \mathrm{mg}$ per os twice daily three weeks before her scheduled repeat cesarean section at 37 weeks of gestation. Her repeat ultrasound showed three cysts, one cyst decreased in size from 6 to 5 centimeters, the other two cysts were stable in size at 3 centimeters, yet nature could not be confirmed. Plan was to omit hydatid cystectomy due to stability of the patient and to continue same dose of Albendazole till delivery. She had smooth cesarean delivery and medical management was stopped two days after delivery. The patient did not manifest any symptoms afterwards.

\section{Conclusion}

In conclusion, hydatid disease is rarely combined with pregnancy. It can be primarily manifested incidentally on obstetrical ultrasound or due to abdominal pain from compression of a gravid uterus. It poses a challenge in regards to management during pregnancy. There are three modalities of management. Medical therapy using Albendazole is advised following the first trimester $[8,14]$ either alone for stable, deep cysts or for those decreasing in size, or in conjunction with intervention aiming to shrink the mass. Percutaneous treatment is used mainly for cysts which do not have daughter cysts. Finally, surgical excision is reserved for ruptured cysts, cysts with biliary fistulae, cysts compressing vital structures, cysts with secondary infection or hemorrhage, or cysts with multiple daughter cysts. Management decision is quite meticulous. It depends on cystic nature and activity and on the stability of the patient. In all cases, cesarean section is advised [8]. In spite of all recommendations, further prospective studies are needed to put clear-cut algorithmic guidelines for hydatid disease management in pregnancy.

\section{References}

1. Ahmed Al-Ani, Abdul-Naser Elzouki, Rashid Mazhar (2013) An Imported Case of Echinococcosis in a Pregnant Lady with Unusual Presentation. Case Reports in Infectious Diseases 2013: 753-848. [crossref]

2. Thompson A, Chiodini PL, Stewart F (2012) Hydatid liver cyst in pregnancy: a case report. British Medical Journal 97: A65.

3. Anandita, Shweta, Bhardwaj S, Sehra A (2015) Pregnancy with Hydatid Cyst of Liver. JCR 5: 542-545.

4. Ghosh JK, Goyal SK, Behera MK, Dixit VK, Jain AK (2014) Hydatid Cyst of Liver Presented as Obstructive Jaundice in Pregnancy; Managed by PAIR. Journal of clinical and experimental Hepatology 4: 366-369. [crossref]

5. Maria A Grácio, António J Santos Grácio (2019) Hydatid Disease and Pregnancy: A Short Note. Integr Gyn Obstet J 2: 1-2.

6. Akbaş A, Daşıran F, Dagmura H, Daldal E, Özsoy Z, et al. (2019) Primary hydatid cyst localized in soft tissue during pregnancy. J Surg Case Rep 2019: rjy324.

7. Ünalp H, Aydin Ç, Yavuzcan A, et al. (2008) Surgical treatment of hepatic hydatic disease during pregnancy: report of two cases. Gynecol Surg 5: 243-245.
8. Celik S, Okyay O, Karaman E, Sert ÖZ, Cim N, et al. (2018) Analysis of factors affecting outcomes of pregnancy complicated by Echinococcus: an algorithm for approach and management. Arch Gynecol Obstet 298: 103-110. [crossref]

9. Pedrosa I, Saíz A, Arrazola J, Ferreirós J, Pedrosa C (2000) Hydatid Disease: Radiologic and Pathologic Features and Complications. Hydatid Disease: Radiologic and Pathologic Features and Complications, The Journal of continuing medical education in radiology 20: 795-817. [crossref]

10. Cennet O, Tirnaksiz MB, Dogrul A, Abbasoglu O (2019) Surgical treatment of cyst hydatid disease of the liver in the era of percutaneous treatment, HPB 21: S568eS676.

11. Sozuer E, Akyuz M, Akbulut S (2014) Open surgery for hepatic hydatid disease. Int Surg 99: 764-769. [crossref]

12. Shams-Ul-Bari, Arif SH, Malik AA, Khaja AR, Dass TA, et al. (2011) Role of albendazole in the management of hydatid cyst liver. Saudi J Gastroenterol 17: 343347. [crossref]

13. Horton RJ (1989) Chemotherapy of Echinococcus infection in man with albendazole. Trans R Soc Trop Med Hyg 83: 97-102. [crossref]

14. Dandan I, Soweid A, Abiad F (2019) Hydatid Cysts. Medscape.

15. Pedro M (2019) Clinical Manifestations and Diagnosis of Echinococcus.

\section{Citation:}

Khazaal J (2021) Hydatid Disease during Pregnancy, the Challenging Management: Case Report and Review of Literature. Integr Gyn Obstet J Volume 4(3): 1-4. 